\title{
ON A CONTINUOUS-STATE POPULATION-SIZE-DEPENDENT BRANCHING PROCESS AND ITS EXTINCTION
}

\author{
YUQIANG LI,* Beijing Normal University
}

\begin{abstract}
A continuous-state population-size-dependent branching process $\left\{X_{t}\right\}$ is a modification of the Jiřina process. We prove that such a process arises as the limit of a sequence of suitably scaled population-size-dependent branching processes with discrete states. The extinction problem for the population $X_{t}$ is discussed, and the limit distribution of $X_{t} / t$ obtained when $X_{t}$ tends to infinity.
\end{abstract}

Keywords: Population-size-dependent branching process; finite-dimensional distribution; convergence; extinction; limit distribution

2000 Mathematics Subject Classification: Primary 60J80

Secondary $60 \mathrm{~J} 05$

\section{Introduction}

A time-homogeneous, nonnegative integer-valued Markov chain $\{X(t), t=0,1, \ldots\}$ is called a population-size-dependent branching process (PSDBP) with discrete states if its transition probabilities are given by

$$
\mathrm{E}\left[s^{X(t+1)} \mid X(t)=k\right]=f(k, s)^{k}, \quad k=0,1, \ldots,
$$

where $0 \leq s \leq 1$ and

$$
f(k, s)=\sum_{j=0}^{\infty} k_{k} p_{j} s^{j}, \quad \text { with }{ }_{k} p_{j} \geq 0 \text { such that } \sum_{j=0}^{\infty} k_{k} p_{j}=1, \quad k=0,1, \ldots,
$$

is a family of probability generating functions governing the reproduction (see, e.g. [3] and [8]). In this paper, we call $f(k, s)$ the reproduction generating function of $\{X(t)\}$. Compared with the classical Galton-Watson process (see, e.g. [1]), the PSDBP takes into account the fact that the reproductive behavior may depend on the current size of the population. This process has been studied by a number of authors and has been applied in a variety of contexts. In a series of papers, Klebaner [8], [9], [10] studied the extinction problem of the process and the convergence of $X(t) / C(t)$ as $t \rightarrow \infty$, for suitable normalizing constants $C(t)$. Jagers [4] gave sufficient conditions for exponent growth for general branching processes, and as a corollary obtained the corresponding results for the PSDBP. Lu and Jagers [15] and Wang [18], [19] studied PSDBPs in random environments. See [5], [7], and [11] for more results on the discrete-state PSDBP.

Received 14 June 2005; revision received 11 August 2005.

* Postal address: School of Mathematical Sciences, Beijing Normal University, Beijing, 100875, P. R. China. Email address: y_q_li@163.com 
In this paper, we study a continuous-state version of the PSDBP. Let

$$
\left\{X_{n}(t), t=0,1, \ldots\right\}
$$

be a sequence of PSDBPs. We prove that, under suitable assumptions, the finite-dimensional distributions of $\left\{X_{n}(t) / n\right\}, n=1,2, \ldots$, converge to those of a $[0, \infty)$-valued Markov process $\{Y(t)\}$, as $n \rightarrow \infty$. We also discuss the extinction problem for $\{Y(t)\}$ and the limiting behavior of $Y(t) / t$ as $t \rightarrow \infty$. The process $\{Y(t)\}$ is the so-called continuous-state PSDBP, defined as follows.

A time-homogeneous Markov process $\{Y(t), t=0,1, \ldots\}$ with state space $[0, \infty)$ is called a continuous-state PSDBP if the Laplace transform of its one-step transition probability $P(x, \mathrm{~d} y)$ is given by

$$
\int_{[0, \infty)} \mathrm{e}^{-\lambda y} P(x, \mathrm{~d} y)=\exp \{-x F(x, \lambda)\},
$$

where

$$
F(x, \lambda)=\beta(x) \lambda+\int_{(0, \infty)}\left(1-\mathrm{e}^{-\lambda u}\right) v(x, \mathrm{~d} u),
$$

with $\beta(x)$ a nonnegative Borel function on $[0, \infty)$ and $(1 \wedge s) v(x, \mathrm{~d} s)$ a finite kernel from $[0, \infty)$ to $(0, \infty)$. In particular, if $\beta(x) \equiv \beta$ and $v(x, \mathrm{~d} s) \equiv v(\mathrm{~d} s)$ are both independent of $x \in[0, \infty)$, the process $\{Y(t)\}$ degenerates to the classical Jiřina process (see, e.g. [6] and [17]). Thus, we can regard $\{Y(t)\}$ as a modification of the Jiřina process whose reproductive behavior depends on the size of the population. Obviously, for each $x \in[0, \infty)$,

$$
\int_{[0, \infty)} \mathrm{e}^{-\lambda y} Q(x, \mathrm{~d} y)=\exp \{-F(x, \lambda)\},
$$

defines an infinitely divisible probability measure $Q(x, \mathrm{~d} y)$. In view of (1.1) and (1.3), we have

$$
P(x, \cdot)=Q(x, \cdot)^{x},
$$

where the right-hand side denotes the $x$-fold convolution introduced in [16, p. 35]. We shall call the function $F(x, \lambda)$ defined by (1.2) the reproduction cumulative function (RCF) of $\{Y(t)\}$.

The continuous-state PSDBP can be used to model a number of biological situations and chemical reactions. For example, for some bacteria whose reproduction depends on their concentration in the medium, it is suitable to describe the change of the concentration using a continuous-state PSDBP. See [9], [12], and the references therein for more examples.

In the remainder of the paper, we write $\mathbb{N}=\{0,1, \ldots\}$ and $\mathbb{N}_{+}=\{1,2, \ldots\}$. For each $n \in \mathbb{N}_{+}$, let $\left\{X_{n}(t)\right\}_{t \in \mathbb{N}}$ be a discrete-state PSDBP with reproduction generating function $f_{n}(k, s)$. Then $Y_{n}(t)=n^{-1} X_{n}(t)$ defines a Markov chain $\left\{Y_{n}(t)\right\}_{t \in \mathbb{N}}$ on $\mathbb{Q}_{n}:=\{k / n, k \in \mathbb{N}\}$. For any $i_{n} \in \mathbb{Q}_{n}$, let

$$
F_{n}\left(i_{n}, \lambda\right)=n\left[1-f_{n}\left(n i_{n}, 1-\lambda / n\right)\right], \quad \lambda \leq n .
$$

In Section 2, we show that the continuous-state PSDBP arises as the limit of a sequence of suitably scaled PSDBPs with discrete states. In Section 3, we discuss the extinction problem and prove a limit theorem for the continuous-state PSDBP. 


\section{The sequential limit theorems of the discrete-state PSDBP}

In this section, we discuss the relation between the discrete-state PSDBP and the continuousstate PSDBP (see Theorems 2.1-2.2). At the end of the section we give two examples to illustrate the theorems.

Proposition 2.1. Let $F_{n}(x, \lambda)$ be defined by (1.4) and let $F(x, \lambda)$ be a continuous function of $(x, \lambda) \in[0, \infty) \times[0, \infty)$. Suppose that

(A1) for any $i_{n}, \mathbb{Q}_{n} \ni i_{n} \rightarrow x$, we have $F_{n}\left(i_{n}, \lambda\right) \rightarrow F(x, \lambda)$ as $n \rightarrow \infty$.

Then $F(x, \lambda)$ is the RCF of a continuous-state PSDBP.

Proof. It is sufficient to prove that $F(x, \lambda)$ has the representation (1.2). Since $F(x, 0)=$ $\lim _{n \rightarrow \infty} F_{n}\left(i_{n}, 0\right)=0$, for any $x \in[0, \infty)$ we may apply Corollary 2 of [13] to obtain the representation

$$
F(x, \lambda)=\int_{[0, \infty)}\left(1-\mathrm{e}^{-\lambda u}\right)\left(1-\mathrm{e}^{-u}\right)^{-1} G(x, \mathrm{~d} u),
$$

where $G(x, \mathrm{~d} u)$ is a finite measure on $[0, \infty)$ and the value of the integrand at $u=0$ is defined as $\lambda$. Clearly, the representation (2.1) can be written as (1.2) with $\beta(x)=G(x,\{0\}) \geq 0$ and $v(x, \mathrm{~d} u)=\left(1-\mathrm{e}^{-u}\right)^{-1} G(x, \mathrm{~d} u)$. It follows that

$$
\int_{(0, \infty)}(1 \wedge u) v(x, \mathrm{~d} u)<\infty .
$$

By the continuity assumption on $F(x, \lambda)$, the measure $G(x, \cdot)$ depends continuously on $x \in$ $[0, \infty)$, by the weak convergence topology. In particular, $G(x, \mathrm{~d} u)$ is a kernel from $[0, \infty)$ to $[0, \infty)$. Then $\beta(\cdot)$ is a measurable function on $[0, \infty)$ and $v(x, \mathrm{~d} u)$ is a kernel from $[0, \infty)$ to $(0, \infty)$.

Lemma 2.1. Under the conditions of Proposition 2.1, assume in addition that

(A2) for any $i_{n}, \mathbb{Q}_{n} \ni i_{n} \rightarrow x$, we have $f_{n}^{\prime}\left(n i_{n}, 1-\right) / n \rightarrow 0$ as $n \rightarrow \infty$, where $f_{n}^{\prime}(k, s)=$ $\mathrm{d} f_{n}(k, s) / \mathrm{d} s$.

Then

$$
H_{n}\left(i_{n}, \lambda\right):=-n \log f_{n}\left(n i_{n}, \mathrm{e}^{-\lambda / n}\right) \rightarrow F(x, \lambda)
$$

as $n \rightarrow \infty$.

Proof. The assumption implies that $f_{n}\left(n i_{n}, 1-\lambda / n\right) \rightarrow 1$. By the mean value theorem, we have

$$
\begin{aligned}
0 & \leq\left|H_{n}\left(i_{n}, \lambda\right)-n \log f_{n}\left(n i_{n}, 1-\lambda / n\right)\right| \\
& \leq \frac{n\left|f_{n}\left(n i_{n}, \mathrm{e}^{-\lambda / n}\right)-f_{n}\left(n i_{n}, 1-\lambda / n\right)\right|}{f_{n}\left(n i_{n}, 1-\lambda / n\right)} \\
& \leq \frac{n f_{n}^{\prime}\left(n i_{n}, 1-\right)\left|\mathrm{e}^{-\lambda / n}-1+\lambda / n\right|}{f_{n}\left(n i_{n}, 1-\lambda / n\right)},
\end{aligned}
$$

where the right-hand side goes to 0 because $f_{n}^{\prime}\left(n i_{n}, 1-\right) / n \rightarrow 0$. Consequently,

$$
\left|H_{n}\left(i_{n}, \lambda\right)-n \log f_{n}\left(n i_{n}, 1-\lambda / n\right)\right| \rightarrow 0 .
$$


By using a Taylor expansion, we find that there is a constant $\theta_{n} \in\left(f_{n}\left(n i_{n}, 1-\lambda / n\right), 1\right)$ such that

$$
\log f_{n}\left(n i_{n}, 1-\lambda / n\right)=f_{n}\left(n i_{n}, 1-\lambda / n\right)-1-\frac{1}{2} \theta_{n}^{-2}\left[f_{n}\left(n i_{n}, 1-\lambda / n\right)-1\right]^{2} .
$$

It follows that

$$
\lim _{n \rightarrow \infty} n \log f_{n}\left(n i_{n}, 1-\lambda / n\right)=\lim _{n \rightarrow \infty} n\left[f_{n}\left(n i_{n}, 1-\lambda / n\right)-1\right]=-F(x, \lambda)
$$

By combining (2.3) with (2.4) we obtain the desired result.

Theorem 2.1. Suppose that conditions (A1) and (A2) hold, and that the distribution of $Y_{n}(0)$ converges weakly to some distribution $\mu$ on $[0, \infty)$ as $n \rightarrow \infty$. The finite-dimensional distributions of $\left\{Y_{n}(t)\right\}$ then converge to those of a continuous-state PSDBP $\{Y(t)\}$ with RCF $F(x, \lambda)$ and initial distribution $\mu$.

Proof. By the Kolmogorov existence theorem, we can construct a Markov process $\{Y(t)\}_{t \in \mathbb{N}}$ on $[0, \infty)$ with initial distribution $\mu$ and one-step transition probability $P(x, \mathrm{~d} y)$ characterized by (1.1). Let $\stackrel{\text { D }}{\rightarrow}$ ' denote convergence in distribution. By assumption, we have $Y_{n}(0) \stackrel{\mathrm{D}}{\rightarrow} Y(0)$. In the sequel, we assume that $\left(Y_{n}(0), \ldots, Y_{n}(m)\right) \stackrel{\mathrm{D}}{\rightarrow}(Y(0), \ldots, Y(m))$ and prove that

$$
\left(Y_{n}(0), \ldots, Y_{n}(m+1)\right) \stackrel{\mathrm{D}}{\rightarrow}(Y(0), \ldots, Y(m+1)),
$$

from which the theorem follows by induction.

It is sufficient to show that, for every $\lambda_{i} \geq 0, i=0,1, \ldots, m+1$,

$$
\mathrm{E}\left[\exp \left\{-\sum_{i=0}^{m+1} \lambda_{i} Y_{n}(i)\right\}\right] \rightarrow \mathrm{E}\left[\exp \left\{-\sum_{i=0}^{m+1} \lambda_{i} Y(i)\right\}\right]
$$

for every $\lambda_{i} \geq 0$. Since $Y_{n}(t)=X_{n}(t) / n$, for any $i_{n} \in \mathbb{Q}_{n}$ we have

$$
\begin{aligned}
\mathrm{E}[\exp & \left.\left\{-\lambda_{m+1} Y_{n}(m+1)\right\} \mid Y_{n}(m)=i_{n}\right] \\
& =\mathrm{E}\left[\exp \left\{-\lambda_{m+1} X^{(n)}(m+1) / n\right\} \mid X_{n}(m)=n i_{n}\right] \\
& =\left[f_{n}\left(n i_{n}, \mathrm{e}^{-\lambda_{m+1} / n}\right)\right]^{n i_{n}} \\
& =\exp \left\{-i_{n} H_{n}\left(i_{n}, \lambda_{m+1}\right)\right\}
\end{aligned}
$$

where $H_{n}(\cdot, \cdot)$ is defined by (2.2). Let $\mathcal{F}_{m}^{(n)}=\sigma\left(\left\{Y_{n}(0), Y_{n}(1), \ldots, Y_{n}(m)\right\}\right)$. From (2.6) and the Markov property, we obtain

$$
\mathrm{E}\left[\exp \left\{-\sum_{i=0}^{m+1} \lambda_{i} Y_{n}(i)\right\}\right]=\mathrm{E}\left[\exp \left\{-\sum_{i=0}^{m} \lambda_{i} Y_{n}(i)\right\} \exp \left\{-Y_{n}(m) H_{n}\left(Y_{n}(m), \lambda_{m+1}\right)\right\}\right] .
$$

Since $\left(Y_{n}(0), \ldots, Y_{n}(m)\right) \stackrel{\mathrm{D}}{\rightarrow}(Y(0), \ldots, Y(m))$, we can use the Skorokhod representation theorem (see [2, p. 102]) to construct random variables $\left(Z_{n}(0), \ldots, Z_{n}(m)\right)$ and $(Z(0), \ldots, Z(m))$ such that

$$
\begin{gathered}
\left(Z_{n}(0), \ldots, Z_{n}(m)\right) \stackrel{\mathrm{D}}{=}\left(Y_{n}(0), \ldots, Y_{n}(m)\right), \\
(Z(0), \ldots, Z(m)) \stackrel{\mathrm{D}}{=}(Y(0), \ldots, Y(m)),
\end{gathered}
$$


and $Z_{n}(i) \rightarrow Z(i)$ almost surely (a.s.) for every $i=0,1, \ldots, m$, where ' $=$ ' means the related random variables have identical distributions. Since $\mathrm{P}\left(\left(Y_{n}(0), \ldots, Y_{n}(m)\right) \in \mathbb{Q}_{n}^{m+1}\right)=1$, we have $\mathrm{P}\left(\left(Z_{n}(0), \ldots, Z_{n}(m)\right) \in \mathbb{Q}_{n}^{m+1}\right)=1$. From (2.7) and (2.8), we obtain

$$
\mathrm{E}\left[\exp \left\{-\sum_{i=0}^{m+1} \lambda_{i} Y_{n}(i)\right\}\right]=\mathrm{E}\left[\exp \left\{-\sum_{i=0}^{m} \lambda_{i} Z_{n}(i)\right\} \exp \left\{-Z_{n}(m) H_{n}\left(Z_{n}(m), \lambda_{m+1}\right)\right\}\right] .
$$

By Lemma 2.1 and the dominated convergence theorem, we see that the right-hand side of (2.9) converges to

$$
\begin{aligned}
& \mathrm{E}\left[\exp \left\{-\sum_{i=0}^{m} \lambda_{i} Z(i)\right\} \exp \left\{-Z(m) F\left(Z(m), \lambda_{m+1}\right)\right\}\right] \\
& =\mathrm{E}\left[\exp \left\{-\sum_{i=0}^{m} \lambda_{i} Y(i)\right\} \exp \left\{-Y(m) F\left(Y(m), \lambda_{m+1}\right)\right\}\right] \\
& =\mathrm{E}\left[\exp \left\{-\sum_{i=0}^{m+1} \lambda_{i} Y(i)\right\}\right]
\end{aligned}
$$

where the last equality follows from the Markov property of $\{Y(t)\}$. We thus obtain (2.5).

Proposition 2.2. Let $F(x, \lambda)$ be a continuous $R C F$ defined by (1.2). Suppose that one of the following conditions holds.

(B1) For a sufficiently small $\delta>0$, we have

$$
\int_{(0, \infty)}(\delta \wedge u) v(x, \mathrm{~d} u) \leq \mathrm{e}^{-\beta(x)}
$$

for all $x \in[0, \infty)$.

(B2) The function

$$
x \mapsto \beta(x)+\int_{(0, \infty)}(1 \wedge u) v(x, \mathrm{~d} u)
$$

is bounded on $[0, \infty)$.

Then there exist probability generating functions $\left\{f_{n}(i, \cdot)\right\}$ such that condition $(A 1)$ is satisfied.

Proof. If condition (B1) is satisfied, we let

$$
f_{n}(i, s)=\exp \left\{\beta\left(\frac{i}{n}\right)(s-1)\right\}+\frac{1}{n} \int_{(0, \infty)}\left(\mathrm{e}^{u n(s-1)}-1\right) v\left(\frac{i}{n}, \mathrm{~d} u\right) .
$$

Then $f_{n}(i, 1)=1$ and

$$
\begin{aligned}
f_{n}(i, 0) & =\exp \left\{-\beta\left(\frac{i}{n}\right)\right\}-\frac{1}{n} \int_{(0, \infty)}\left(1-\mathrm{e}^{-u n}\right) v\left(\frac{i}{n}, \mathrm{~d} u\right) \\
& \geq \exp \left\{-\beta\left(\frac{i}{n}\right)\right\}-\frac{1}{n} \int_{(0, \infty)}(1 \wedge u n) v\left(\frac{i}{n}, \mathrm{~d} u\right) \\
& =\exp \left\{-\beta\left(\frac{i}{n}\right)\right\}-\int_{(0, \infty)}\left(n^{-1} \wedge u\right) v\left(\frac{i}{n}, \mathrm{~d} u\right) .
\end{aligned}
$$


It follows that $f_{n}(i, 0) \geq 0$ for sufficiently large $n \geq 1$. On the other hand, $f_{n}(i, s)$ is clearly analytic in $s \in(-1,1)$, with

$$
\frac{\mathrm{d}^{k} f_{n}(i, s)}{\mathrm{d} s^{k}}=\beta\left(\frac{i}{n}\right)^{k} \exp \left\{\beta\left(\frac{i}{n}\right)(s-1)\right\}+n^{k-1} \int_{(0, \infty)} u^{k} \mathrm{e}^{u n(s-1)} v\left(\frac{i}{n}, \mathrm{~d} u\right) \geq 0 .
$$

Thus, $f_{n}(i, s)$ is a probability generating function. Let $F_{n}\left(i_{n}, \lambda\right)$ be defined by (1.4). It is easy to check that

$$
\begin{aligned}
F_{n}\left(i_{n}, \lambda\right) & =n\left(1-\mathrm{e}^{-\beta\left(i_{n}\right) \lambda / n}\right)+\int_{(0, \infty)}\left(1-\mathrm{e}^{-\lambda u}\right) v\left(i_{n}, \mathrm{~d} u\right) \\
& =n\left[1-\beta\left(i_{n}\right) \lambda / n-\mathrm{e}^{-\beta\left(i_{n}\right) \lambda / n}\right]+F\left(i_{n}, \lambda\right),
\end{aligned}
$$

which converges to $F(x, \lambda)$ if $i_{n} \rightarrow x$.

If condition (B2) holds, we choose an integer

$$
K>\sup _{x \in[0, \infty)}\left[\beta(x)+\int_{(0, \infty)}(1 \wedge u) v(x, \mathrm{~d} u)\right]
$$

and let

$$
h_{n}(i, s)=1+\frac{\beta(i / n)}{K}(s-1)+\frac{1}{n K} \int_{(0, \infty)}\left(\mathrm{e}^{n u(s-1)}-1\right) v\left(\frac{i}{n}, \mathrm{~d} u\right) .
$$

It is easy to check that $h_{n}(i, s)$ is a probability generating function and that, if $i_{n} \rightarrow x$,

$$
n\left[1-h_{n}\left(n i_{n}, 1-\frac{\lambda}{n}\right)\right]=\frac{1}{K} F\left(i_{n}, \lambda\right) \rightarrow \frac{1}{K} F(x, \lambda) .
$$

Moreover, $f_{n}(i, s):=\left[h_{n}(i, s)\right]^{K}$ is also a probability generating function. Let $F_{n}\left(i_{n}, \lambda\right)$ again be defined by (1.4). By a Taylor expansion,

$$
F_{n}\left(i_{n}, \lambda\right)=n K\left[1-h_{n}\left(n i_{n}, 1-\lambda / n\right)\right]-\frac{1}{2} n K(K-1) \eta^{K-2}\left[h_{n}\left(n i_{n}, 1-\lambda / n\right)-1\right]^{2},
$$

where $\eta \in\left(h_{n}\left(n i_{n}, 1-\lambda / n\right), 1\right)$. From (2.12) we have

$$
\lim _{n \rightarrow \infty} F_{n}\left(i_{n}, \lambda\right)=\lim _{n \rightarrow \infty} n K\left[1-h_{n}\left(n i_{n}, 1-\lambda / n\right)\right]=F(x, \lambda),
$$

which completes the proof.

Remark 2.1. An analogue of condition (B2) was used in [14] in the study of the convergence of branching particle systems to measure-valued processes. Although this condition is more natural and easier to understand than condition (B1), it is not always satisfied, even in some simple cases, as we will see in Example 2.1. We also remark that if

$$
\sup _{x \in[0, \infty)}\left[\beta(x)+\int_{(0, \infty)} v(x, \mathrm{~d} u)\right]<\infty
$$

then both (B1) and (B2) are satisfied. 
Proposition 2.3. Let $F(x, \lambda)$ be a continuous $R C F$ defined by (1.2), and assume that the conditions of Proposition 2.2 hold. Suppose in addition that

(B3) the function $x \mapsto \int_{(0, \infty)} u v(x, \mathrm{~d} u)$ is bounded on $[0, \infty)$.

Then there exist probability generating functions $\left\{f_{n}(i, \cdot)\right\}$ such that conditions $(A 1)$ and $(A 2)$ are satisfied.

Proof. By Proposition 2.2, there exists a family of probability generating functions $\left\{f_{n}(i, \cdot)\right\}$ such that (A1) holds. If (B1) holds then $f_{n}(i, s)$ is defined by $(2.10)$ and we have

$$
f_{n}^{\prime}\left(n i_{n}, 1-\right)=\beta\left(i_{n}\right)+\int_{(0, \infty)} u v\left(i_{n}, \mathrm{~d} u\right) .
$$

We have the same equality if (B2) holds and $f_{n}(i, s)=h_{n}(i, s)^{K}$, with $h_{n}$ given by (2.11). By (B3) and the continuity of $F(x, \lambda)$, it follows that $f_{n}^{\prime}\left(n i_{n}, 1-\right) / n \rightarrow 0$ for any $i_{n}$ such that $\mathbb{Q}_{n} \ni i_{n} \rightarrow x \in[0, \infty)$.

Theorem 2.1 and Proposition 2.3 imply the following result.

Theorem 2.2. Let $\{Y(t)\}$ be a continuous-state PSDBP with continuous $R C F F(x, \lambda)$ satisfying the conditions of Proposition 2.3. Then there exists a sequence of discrete-state PSDBPs $\left\{X_{n}(t)\right\}$ such that the finite-dimensional distributions of $\left\{X_{n}(t) / n\right\}$ converge to those of $\{Y(t)\}$.

Recall that a Jiřina process is the limit of a sequence of Galton-Watson processes (see, e.g. [6]). Thus, the above theorems generalize the classical result in some sense. In the remainder of this section, we give two examples to illustrate the theorems.

Example 2.1. Let $\left\{X_{n}(t)\right\}_{t \in \mathbb{N}}$ be a sequence of discrete-state PSDBPs with reproduction distributions

$$
{ }_{k} p_{j}^{(n)}=\frac{\mathrm{e}^{-k / n}(k / n)^{j}}{j !}, \quad j \geq 0 .
$$

Then

$$
f_{n}(k, s)=\sum_{j=0}^{\infty} k p_{j}^{(n)} s^{j}=\exp \left\{-\frac{k(1-s)}{n}\right\}
$$

and, as $i_{n} \rightarrow x$, we have

$$
F_{n}\left(i_{n}, \lambda\right):=n\left(1-f_{n}\left(n i_{n}, 1-\lambda / n\right)\right)=n\left[1-\mathrm{e}^{-i_{n} \lambda / n}\right] \rightarrow x \lambda,
$$

and $f_{n}^{\prime}\left(n i_{n}, 1\right) / n=i_{n} / n \rightarrow 0$. Suppose that $X_{n}(0)=n$. Then $Y_{n}(0)=X_{n}(0) / n \equiv 1$, and Theorem 2.1 implies that the finite-dimensional distributions of $\left\{Y_{n}(t)\right\}$ converge to those of a continuous-state PSDBP process $\{Y(t)\}$ with RCF $F(x, \lambda)=\lambda x$ and $Y(0)=1$. Clearly, the limit process $\{Y(t)\}$ is deterministic. It is not hard to see that condition (B1) holds for $\{Y(t)\}$, but condition (B2) does not hold.

Example 2.2. Let $\alpha \in(0,1], \beta>0$, and $\gamma>0$, with $\beta \leq 1+\gamma$. Let $\left\{X_{n}(t)\right\}_{t \in \mathbb{N}}$ be a sequence of discrete-state PSDBPs with reproduction distributions given by

$$
{ }_{k} p_{j}^{(n)}= \begin{cases}1-\frac{\beta \alpha^{k / n}}{1+k \gamma}, & j=0, \\ \frac{\beta \alpha^{k / n}(k \gamma)^{j-1}}{(1+k \gamma)^{j+1}}, & j>0 .\end{cases}
$$


Then

$$
f_{n}(k, s)=\sum_{j=0}^{\infty} k p_{j}^{(n)} s^{j}=1-\frac{\beta \alpha^{k / n}(1-s)}{1+k \gamma-k \gamma s}
$$

If $i_{n} \rightarrow x$, we have

$$
F_{n}\left(i_{n}, \lambda\right)=\frac{\beta \alpha^{i_{n}} \lambda}{1+i_{n} \gamma \lambda} \rightarrow F(x, \lambda):=\frac{\beta \alpha^{x} \lambda}{1+\gamma \lambda x}
$$

and

$$
\frac{f_{n}^{\prime}\left(n i_{n}, 1-\right)}{n}=\frac{\beta \alpha^{i_{n}}}{n} \rightarrow 0 .
$$

By Theorem 2.1, if $Y_{n}(0)$ converges in distribution then the finite-dimensional distributions of $\left\{Y_{n}(t)\right\}$ converge to those of a continuous-state PSDBP with RCF $F(x, \lambda)$. It is not hard to show that $F(x, \lambda)$ has the decomposition (1.2) with $\beta(x)=0$ and

$$
v(x, \mathrm{~d} u)=\frac{\beta \alpha^{x}}{\gamma^{2} x^{2}} \mathrm{e}^{-u / \gamma x} \mathrm{~d} u
$$

for $x>0$.

\section{The extinction of the continuous-state PSDBP}

In this section, we discuss the extinction problem for the continuous-state PSDBP $\left\{Y_{t}\right\}$. Since $\left\{Y_{t}\right\}$ has continuous states and its Laplace transform cannot be expressed explicitly, we cannot infer that $Y_{t}$ tends to either 0 or $\infty$, as we do for the discrete-state PSDBP (see, e.g. [9]) and the classical continuous-state branching processes (see, e.g. [1]). However, we can still find some interesting properties (see Theorems 3.1-3.3) of the extinction, under certain conditions. We can also study the limit distribution of $Y_{t} / t$ as $Y_{t}$ tends to infinity (see Theorem 3.4). Let

Let $\left\{Y_{t}\right\}_{t \in \mathbb{N}}$ be a continuous-state PSDBP with RCF $F(x, \lambda)$ and initial state $Y_{0}=x_{0} \geq 0$.

$$
F^{(k)}(x, \lambda):=\mathrm{d}^{k} F(x, \lambda) / \mathrm{d} \lambda^{k},
$$

and write $m_{k}(x)=(-1)^{k-1} F^{(k)}(x, 0+)$ if the required derivative exists. Obviously, $m_{k}(x) \geq 0$ and

$$
F(x, \lambda) \leq m_{1}(x) \lambda .
$$

Lemma 3.1. Suppose that $m_{k}(x)<M_{k}\left(x^{(k-3) \vee 0} \vee 1\right)$ for all $x>0$ and $k \in \mathbb{N}_{+}$, where $M_{k}$ is a positive constant depending only on $k$. Then, for any $k \geq 3$, there exists a positive constant $A_{k}$ such that

$$
\left|\mathrm{E}\left[\left\{Y_{t+1}-m_{1}\left(Y_{t}\right) Y_{t}\right\}^{k} \mid Y_{t}\right]\right| \leq A_{k}\left(Y_{t}^{k-2} \vee 1\right) .
$$

Proof. We shall prove the inequality by induction on $k$. Note that

$$
\left|\mathrm{E}\left[\left\{Y_{t+1}-m_{1}\left(Y_{t}\right) Y_{t}\right\}^{k} \mid Y_{t}\right]\right|=\left|\frac{\mathrm{d}^{k} \exp \left\{Y_{t}\left(\lambda m_{1}\left(Y_{t}\right)-F\left(Y_{t}, \lambda\right)\right)\right\}}{\mathrm{d} \lambda^{k}}\right|_{\lambda=0+} .
$$

It is easy to show that

$$
\left|\mathrm{E}\left[\left\{Y_{t+1}-m_{1}\left(Y_{t}\right) Y_{t}\right\}^{3} \mid Y_{t}\right]\right|=m_{3}\left(Y_{t}\right) Y_{t}<M_{3} Y_{t} .
$$


Now suppose that (3.2) holds for all $i, 3 \leq i<k$. From (3.3), by tedious calculations, we obtain

$$
\begin{aligned}
\left|\mathrm{E}\left[\left\{Y_{t+1}-m_{1}\left(Y_{t}\right) Y_{t}\right\}^{k} \mid Y_{t}\right]\right| \\
\leq Y_{t} \sum_{j=2}^{k-3}\left(\begin{array}{c}
k-1 \\
j-1
\end{array}\right) m_{j}\left(Y_{t}\right)\left|\mathrm{E}\left[\left\{Y_{t+1}-m_{1}\left(Y_{t}\right) Y_{t}\right\}^{k-j} \mid Y_{t}\right]\right| \\
\quad+Y_{t} m_{k}\left(Y_{t}\right)+\frac{(k-1)(k-2)}{2} Y_{t}^{2} m_{2}\left(Y_{t}\right) m_{k-2}\left(Y_{t}\right) \\
\leq\left(M_{k}+\sum_{j=2}^{k-3}\left(\begin{array}{l}
k-1 \\
j-1
\end{array}\right) M_{j} A_{k-j}+\frac{(k-1)(k-2)}{2} M_{2} M_{k-2}\right)\left(Y_{t}^{k-2} \vee 1\right),
\end{aligned}
$$

where the term containing the summation disappears if $k=4$. Let

$$
A_{k}=M_{k}+\sum_{j=2}^{k-3}\left(\begin{array}{l}
k-1 \\
j-1
\end{array}\right) M_{j} A_{k-j}+\frac{(k-1)(k-2)}{2} M_{2} M_{k-2} .
$$

Then (3.2) follows from (3.4) for $i=k$.

Lemma 3.2. Under the assumption of Lemma 3.1, for any $k \geq 2$ we have

$$
\mathrm{E}\left[Y_{t+1}^{k} \mid Y_{t}\right]=\left(m_{1}\left(Y_{t}\right) Y_{t}\right)^{k}+\left(\begin{array}{l}
k \\
2
\end{array}\right) m_{1}^{k-2}\left(Y_{t}\right) m_{2}\left(Y_{t}\right) Y_{t}^{k-1}+R\left(Y_{t}, k\right),
$$

where

$$
\begin{aligned}
\left|R\left(Y_{t}, k\right)\right| & :=\left|\sum_{i=3}^{k}\left(\begin{array}{l}
k \\
i
\end{array}\right) \mathrm{E}\left[\left\{Y_{t+1}-m_{1}\left(Y_{t}\right) Y_{t}\right\}^{i} \mid Y_{t}\right]\left(m_{1}\left(Y_{t}\right) Y_{t}\right)^{k-i}\right| \\
& <B_{k}\left(Y_{t}^{k-2} \vee 1\right), \text { for some } B_{k}>0 .
\end{aligned}
$$

Proof. When $k=2$, we obtain (3.5) from

$$
\mathrm{E}\left[Y_{t+1}^{2} \mid Y_{t}\right]=m_{1}^{2}\left(Y_{t}\right) Y_{t}^{2}+m_{2}\left(Y_{t}\right) Y_{t}
$$

Note that, for $k>2$,

$$
\begin{aligned}
\mathrm{E}\left[Y_{t+1}^{k} \mid Y_{t}\right] & =\mathrm{E}\left[\left\{Y_{t+1}-m_{1}\left(Y_{t}\right) Y_{t}+m_{1}\left(Y_{t}\right) Y_{t}\right\}^{k} \mid Y_{t}\right] \\
& =\left(m_{1}\left(Y_{t}\right) Y_{t}\right)^{k}+\left(\begin{array}{l}
k \\
2
\end{array}\right) m_{1}^{k-2}\left(Y_{t}\right) m_{2}\left(Y_{t}\right) Y_{t}^{k-1}+R\left(Y_{t}, k\right) .
\end{aligned}
$$

Equations (3.2) and (3.6) imply that

$$
\left|R\left(Y_{t}, k\right)\right| \leq \sum_{i=3}^{k}\left(\begin{array}{l}
k \\
i
\end{array}\right) A_{i} M_{1}^{k-i}\left(Y_{t}^{i-2} \vee 1\right) Y_{t}^{k-i} \leq\left(Y_{t}^{k-2} \vee 1\right) \sum_{i=3}^{k}\left(\begin{array}{l}
k \\
i
\end{array}\right) A_{i} M_{1}^{k-i}
$$

By combining (3.7) with (3.8), we recover (3.5) with

$$
B_{k}=\sum_{i=3}^{k}\left(\begin{array}{l}
k \\
i
\end{array}\right) A_{i} M_{1}^{k-i}
$$

for all $k>1$. 
Theorem 3.1. If $\sup _{x \in(0, \infty)} m_{1}(x)<1$ or $m_{1}(x) \nearrow 1$ as $x \rightarrow \infty$, then $Y_{t} \rightarrow 0$ a.s.

Proof. Note that

$$
\mathrm{E}\left[Y_{t+1} \mid Y_{t}\right]=Y_{t} m_{1}\left(Y_{t}\right) \leq Y_{t} .
$$

By the supermartingale convergence theorem, we know there exists a nonnegative integrable random variable $Y$ such that

$$
Y_{t} \rightarrow Y \quad \text { a.s. }
$$

Consider the following two cases.

Case 1. $\sup _{x \in(0, \infty)} m_{1}(x)<1$. Let $m=\sup _{x \in(0, \infty)} m_{1}(x)<1$. For any $\lambda \geq 0$, by (3.1) and the dominated convergence theorem we have

$$
\begin{aligned}
1 \geq \mathrm{E}\left[\mathrm{e}^{-\lambda Y}\right] & =\lim _{t \rightarrow \infty} \mathrm{E}\left[\mathrm{e}^{-\lambda Y_{t+1}}\right]=\lim _{t \rightarrow \infty} \mathrm{E}\left[\mathrm{E}\left[\mathrm{e}^{-\lambda Y_{t+1}} \mid Y_{t}\right]\right]=\lim _{t \rightarrow \infty} \mathrm{E}\left[\mathrm{e}^{-Y_{t} F\left(Y_{t}, \lambda\right)}\right] \\
& \geq \lim _{t \rightarrow \infty} \mathrm{E}\left[\mathrm{e}^{-m Y_{t} \lambda}\right] \geq \lim _{t \rightarrow \infty} \mathrm{E}\left[\mathrm{e}^{-m^{2} Y_{t-1} \lambda}\right] \geq \cdots \geq \lim _{t \rightarrow \infty} \mathrm{E}\left[\mathrm{e}^{-x_{0} m^{t+1} \lambda}\right]=1,
\end{aligned}
$$

which implies that $Y=0$ a.s.

Case 2. $m_{1}(x) \nearrow 1$. There exists a $\delta(x)>0$ such that $m_{1}(x)+\delta(x) \nearrow 1$. From (3.9) we find that

$$
\prod_{i=0}^{n-1}\left[m_{1}\left(Y_{i}\right)+\delta\left(Y_{i}\right)\right] \rightarrow 0 \quad \text { a.s. }
$$

as $n \rightarrow \infty$. Let

$$
X_{t}=\frac{Y_{t}}{\prod_{i=0}^{t-1}\left[m_{1}\left(Y_{i}\right)+\delta\left(Y_{i}\right)\right]} .
$$

Obviously, $X_{t}$ is a nonnegative supermartingale. There thus exists an integrable random variable $X$ such that $X_{t} \rightarrow X$ a.s. Hence, $Y_{t}=X_{t} \prod_{i=0}^{t-1}\left[m_{1}\left(Y_{i}\right)+\delta\left(Y_{i}\right)\right] \rightarrow 0$ a.s.

Theorem 3.2. (i) Suppose that $\bar{m}:=\inf _{x \in(0, \infty)} m_{1}(x)>1$ and $m_{2}(x)<M$ for some $M>0$. Then $Y_{t} \rightarrow Y \in[0, \infty]$ a.s.

(ii) If $\beta:=\inf _{x \in(0, \infty)} \beta(x)>1$ and $x_{0}>0$, then $Y_{t} \rightarrow \infty$ a.s.

(iii) If $\bar{\beta}:=\sup _{x \in(0, \infty)} \beta(x)<1$ and, as $\lambda \rightarrow \infty$,

$$
\sup _{x \in(0, \infty)} \int_{(0, \infty)}\left(\frac{1}{\lambda} \wedge u\right) v(x, \mathrm{~d} u) \rightarrow 0,
$$

then there exist an $r, 0<r<1$, and a $\lambda_{0}, \lambda_{0}>0$, such that, for any $\lambda \geq \lambda_{0}$, we have

$$
F(x, \lambda) \leq r \lambda .
$$

(iv) If, for $\lambda \geq \lambda_{0}>0, F(x, \lambda)$ satisfies (3.11), then $Y_{t} \rightarrow Y \in[0, \infty]$ a.s.

Proof. (i) Since $F^{(3)}(x, \lambda)>0$, by a Taylor expansion it is easy to show that

$$
F(x, \lambda) \geq\left(m_{1}(x)-\frac{1}{2} m_{2}(x) \lambda\right) \lambda .
$$

By combining (3.12) with the assumptions, we obtain

$$
F(x, \lambda) \geq \alpha \lambda,
$$


for a sufficiently small $\lambda>0$ and some constant $\alpha>1$. From (3.13), we have

$$
\mathrm{E}\left[\mathrm{e}^{-\lambda Y_{t+1}} \mid Y_{t}\right]=\mathrm{e}^{-Y_{t} F\left(Y_{t}, \lambda\right)} \leq \mathrm{e}^{-\alpha \lambda Y_{t}} \leq \mathrm{e}^{-\lambda Y_{t}} .
$$

Thus, $\left\{\mathrm{e}^{-\lambda Y_{t}}\right\}$ is a nonnegative supermartingale. There exists a nonnegative random variable $X \in[0,1]$ such that $\mathrm{e}^{-\lambda Y_{t}} \rightarrow X$ a.s. Hence, $Y_{t} \rightarrow Y=-\lambda^{-1} \log X \in[0, \infty]$ a.s.

(ii) Note that $F(x, \lambda) \geq \beta(x) \lambda \geq \beta \lambda$. By an argument similar to that used in proving (i), we have

$$
Y_{n} \rightarrow Y \in[0, \infty] \text { a.s. }
$$

Now note that, for $\lambda>0$,

$$
\begin{aligned}
0 \leq \mathrm{E}\left[\mathrm{e}^{-\lambda Y}\right] & =\lim _{t \rightarrow \infty} \mathrm{E}\left[\mathrm{e}^{-\lambda Y_{t+1}}\right]=\lim _{t \rightarrow \infty} \mathrm{E}\left[\mathrm{E}\left[\mathrm{e}^{-\lambda Y_{t+1}} \mid Y_{t}\right]\right]=\lim _{t \rightarrow \infty} \mathrm{E}\left[\mathrm{e}^{-Y_{t} F\left(Y_{t}, \lambda\right)}\right] \\
& \leq \lim _{t \rightarrow \infty} \mathrm{E}\left[\mathrm{e}^{-\lambda Y_{t} \beta}\right] \leq \lim _{t \rightarrow \infty} \mathrm{E}\left[\mathrm{e}^{-\lambda Y_{t-1} \beta^{2}}\right] \leq \cdots \leq \lim _{t \rightarrow \infty} \mathrm{E}\left[\mathrm{e}^{-x_{0} \beta^{t+1} \lambda}\right]=0 .
\end{aligned}
$$

Thus, $Y=\infty$ a.s.

(iii) Since $\bar{\beta}<1$, there exists a $\delta_{1}>0$ such that $\bar{\beta}+\delta_{1}<1$. From (3.10), there exists a $\lambda_{0}>0$ such that, for $\lambda \geq \lambda_{0}$,

$$
\sup _{x \in(0, \infty)} \int_{(0, \infty)}\left(\frac{1}{\lambda} \wedge u\right) v(x, \mathrm{~d} u)<\delta_{1} .
$$

Thus, (3.11) holds for $\lambda \geq \lambda_{0}$.

(iv) Suppose that (3.11) holds for $\lambda \geq \lambda_{0}>0$. Then

$$
\mathrm{E}\left[\mathrm{e}^{-\lambda_{0} Y_{t+1}} \mid Y_{t}\right]=\mathrm{e}^{-Y_{t} F\left(Y_{t}, \lambda_{0}\right)} \geq \mathrm{e}^{-r \lambda_{0} Y_{t}} \geq \mathrm{e}^{-\lambda_{0} Y_{t}} .
$$

Hence, there exists a random variable $Y \in[0, \infty]$ such that $Y_{t} \rightarrow Y$ a.s.

The main ideas of the following two theorems come from [9].

Theorem 3.3. If, for any $x \geq 0$,

$$
\int_{0}^{\infty} \mathrm{e}^{-x F(x, \lambda)-\lambda} \mathrm{d} \lambda \leq \frac{1}{x+1},
$$

then there exists a random variable $Y$ such that $Y_{t} \rightarrow Y$ a.s. and $\mathrm{P}\{Y=0\} \leq 1 /\left(x_{0}+1\right)$.

Proof. The proof is similar to that of Theorem 1.2 of [9]. We omit it here.

Remark 3.1. Theorem 3.1 and Theorem 3.2 discuss extinction for $m_{1}(x)<1$ and $m_{1}(x)>1$, respectively. Theorem 3.3 gives a nontrivial upper bound for the extinction probability.

In the following we will consider the distribution of $\lim _{t \rightarrow \infty} Y_{t} / t$ when $m_{1}(x) \rightarrow 1$ as $x \rightarrow \infty$ (with $m_{1}(x)>1$ ) and $Y_{t} \rightarrow \infty$ as $t \rightarrow \infty$. To this end we let $\varepsilon(x)=m_{1}(x)-1$ and consider the following two conditions:

$$
\begin{gathered}
x \varepsilon(x)=c+\eta(x), \quad 0<c<\infty, \quad \lim _{x \rightarrow \infty} \eta(x)=0, \\
\lim _{x \rightarrow \infty} m_{2}(x)=\sigma^{2}, \quad 0<\sigma<\infty .
\end{gathered}
$$


Theorem 3.4. Suppose that (E1) and (E2) hold. Under the assumption of Lemma 3.1, if $Y_{t} \rightarrow \infty$ a.s., then the distribution of $Y_{t} / t$ converges to the $\Gamma(r, \alpha)$ distribution on $[0, \infty)$, where $\alpha=2 / \sigma^{2}$ and $r=2 c / \sigma^{2}$; that is,

$$
\lim _{t \rightarrow \infty} \mathrm{P}\left\{Y_{t} / t \leq x\right\}=\int_{0}^{x} \frac{\alpha^{r}}{\Gamma(r)} s^{r-1} \mathrm{e}^{-\alpha s} \mathrm{~d} s .
$$

Proof. The proof is very similar to that of Theorem 1.5 of [9]. In the following we emphasize the differences.

By the method of moments, we shall prove that

$$
\beta_{k}=\lim _{n \rightarrow \infty} \mathrm{E}\left[\left(Y_{t} / t\right)^{k}\right]<\infty
$$

with $\beta_{1}=c$ and, for $k>1$,

$$
\beta_{k}=\left(c+\frac{1}{2}(k-1) \sigma^{2}\right) \beta_{k-1} .
$$

We will use induction on $k$. For $k=1$, by starting from (E1) and iterating, we find that

$$
\mathrm{E}\left[Y_{t}\right]=x_{0}+t c+\sum_{i=0}^{t-1} \mathrm{E}\left[\eta\left(Y_{i}\right)\right]
$$

From (3.14) it is easy to obtain $\beta_{1}=\lim _{t \rightarrow \infty} \mathrm{E}\left[Y_{t} / t\right]=c$. Now suppose that, for all $i \leq k-1$, $\beta_{i}=\lim _{t \rightarrow \infty} \mathrm{E}\left[\left(Y_{t} / t\right)^{i}\right]<\infty$. Let $\alpha_{t}^{(k)}=\mathrm{E}\left[Y_{t}^{k}\right]$ and $\gamma(x)=k \eta(x)+\left(\begin{array}{c}k \\ 2\end{array}\right)\left(\sigma^{2}-m_{2}(x)\right)$. Then, by Lemma 3.2 and the argument in the proof of Theorem 1.5 of [9], we have

$$
\alpha_{t+1}^{(k)}=\alpha_{t}^{(k)}+\left[k c+\left(\begin{array}{l}
k \\
2
\end{array}\right) \sigma^{2}\right] \alpha_{t}^{(k-1)}+\mathrm{E}\left[Y_{t}^{k-1} \gamma\left(Y_{t}\right)\right]+\mathrm{E}\left[D_{t, k}\right],
$$

where $D_{t, k}$ is a random variable such that $\left|D_{t, k}\right| \leq C_{k}\left(Y_{t}^{k-2} \vee 1\right)$ for some positive constant $C_{k}$. We point out that, in our case,

$$
0 \leq \lim _{t \rightarrow \infty} \mathrm{E}\left[\left|D_{t, k} / t^{k-1}\right|\right] \leq C_{k} \lim _{t \rightarrow \infty}\left(\mathrm{E}\left[Y_{t}^{k-2} / t^{k-1}\right]+1 / t^{k-1}\right)=0 .
$$

The remainder of the proof is similar to that of Theorem 1.5 of [9].

\section{Acknowledgements}

The author is greatly indebted to Professor Zenghu Li for his supervision. Thanks are also due to the referee for helpful comments.

\section{References}

[1] Athreya, K. B. ANd Ney, P. (1972). Branching Processes. Springer, Berlin.

[2] Ethier, S. N. And Kurtz, T. (1986). Markov Processes. Characterization and Convergence. John Wiley, New York.

[3] Höpfner, R. (1985). On some classes of population-size-dependent Galton-Watson processes. J. Appl. Prob. 22, 25-36.

[4] Jagers, P. (1996). Population-size-dependent branching processes. J. Appl. Math. Stoch. Analysis 9, $449-457$.

[5] Jagers, P. and Klebaner, F. C. (2000). Population-size-dependent and age-dependent branching processes. Stoch. Process. Appl. 87, 235-254.

[6] JIŘInA, M. (1960). Stochastic branching processes with continuous state space. Czech. Math. J. 8, $292-313$. 
[7] Klebaner, F. C. (1983). Population-size-dependent branching processes with linear rate of growth. J. Appl. Prob. 20, 242-250.

[8] Klebaner, F. C. (1984). Geometric rate of growth in population-size-dependent branching processes. J. Appl. Prob. 21, 40-49.

[9] Klebaner, F. C. (1984). On population-size-dependent branching processes. Adv. Appl. Prob. 16, 30-55.

[10] Klebaner, F. C. (1985). A limit theorem for population-size-dependent branching processes. J. Appl. Prob. 22, 48-57.

[11] Klebaner, F. C. (1993). Population-size-dependent branching processes with a threshold. Stoch. Process. Appl. 46, 115-127.

[12] LabKovsKiI, V. (1972). A limit theorem for generalized branching random processes depending on the size of the population. Theory Prob. Appl. 17, 72-85.

[13] Li, Z. H. (1991). Integral representations of continuous functions. Chinese Sci. Bull. 36, 979-983.

[14] LI, Z. H. (1992). Measure-valued branching processes with immigration. Stoch. Process. Appl. 43, $249-264$.

[15] LU, Z. W. AND JAGERS, P. (2004). A note on the asymptotic behaviour of the extinction probability in supercritical population-size-dependent branching process with independent and identically distributed random environments. J. Appl. Prob. 41, 176-186.

[16] Sato, K.-I. (1999). Lévy Processes and Infinitely Divisible Distributions. Cambridge University Press.

[17] Seneta, E. AND Vere-Jones, D. (1969). On a problem of M. Jiřina concerning continuous state branching processes. Czech. Math. J. 19, 277-283.

[18] WANG, H. X. (1999). Extinction of population-size-dependent branching processes in random environments. $J$. Appl. Prob. 36, 146-154.

[19] Wang, H. X. And DaI, Y. (1998). Population-size-dependent branching processes in Markovian random environments. Chinese Sci. Bull. 43, 635-638. 